\section{Distributed optical fibre sensors based on spontaneous Brillouin scattering employing multimode Fabry-Pérot lasers}

\section{M.A. Soto, G. Bolognini and F. Di Pasquale}

Proposed and characterised is the use of multi-longitudinal mode Fabry-Pérot laser sources, in distributed optical fibre sensors based on spontaneous Brillouin scattering, for simultaneous strain and temperature measurements. Experimental results show that, by using such multi-longitudinal mode lasers, it is possible to measure the Brillouin spectrum, even if the overall laser spectral linewidth is larger than the Brillouin frequency shift. In this case, a suitable receiver scheme, combining optical and electrical heterodyne detection, is needed. Results report an increase in the threshold value of the input peak power before onset of nonlinear effects, allowing for a better sensing performance compared to single-longitudinal mode lasers.

Introduction and theory: Distributed Brillouin-based optical fibre sensors have been widely reported in recent years owing to their possibilities to perform distributed strain and temperature sensing along an optical fibre [1, 2]. In particular, sensors based on stimulated Brillouin scattering (SBS) require access to both fibre-ends and the use of two optical fibres to distinguish between strain and temperature effects, owing to the strain-temperature cross-sensitivity of the Brillouin gain [1]. On the other hand, sensors based on spontaneous Brillouin scattering (SpBS) only need access to a single fibre-end and allow for simultaneous strain-temperature measurements along the same optical fibre by measuring simultaneously the SpBS intensity and frequency shift (BFS) [2].

In this Letter we propose and characterise the use of multi-longitudinal mode (also called multimode) lasers to improve the sensing performance of SpBSbased sensors. Specifically, in our experiments we have used a Fabry-Perot (FP) laser centred at $\sim 1550 \mathrm{~nm}$, which is among the most common laser sources used in optical communications. We show that with such multimode lasers we can increase the maximum input power threshold value before the onset of fibre nonlinearities, enhancing the sensor performance. In addition, this technique can be efficiently combined with the use of optical pulse coding techniques for further performance enhancement [3].

In SpBS-based sensors the $\operatorname{SpBS}$ intensity $\left(\Delta P_{B}\right)$ and $\operatorname{BFS}\left(\Delta v_{B}\right)$ are both linearly dependent on strain and temperature; this makes the simultaneous strain $(\Delta \varepsilon)$ and temperature $(\Delta T)$ sensing along a single optical fibre possible by using the following matrix relation:

$$
\left[\begin{array}{c}
\Delta v_{B} \\
\Delta P_{B}
\end{array}\right]=\left[\begin{array}{ll}
C_{v_{B} \varepsilon} & C_{v_{B} T} \\
C_{P_{B} \varepsilon} & C_{P_{B} T}
\end{array}\right]\left[\begin{array}{c}
\Delta \varepsilon \\
\Delta T
\end{array}\right]
$$

where $C_{v B \varepsilon}=0.046 \mathrm{MHz} / \mu \varepsilon, C_{v B T}=1.07 \mathrm{MHz} /{ }^{\circ} \mathrm{C}, C_{P B_{s}}=8 \times 10^{-4}$ $\% / \mu \varepsilon$, and $C_{P B T}=0.36 \% / \mathrm{C}$ are the strain and temperature coefficients for BFS and SpBS power [2].

The performance of SpBS-based sensors is essentially limited by the onset of nonlinear optical effects, most notably SBS, which imposes the maximum peak power allowed at fibre input. We have shown that optical pulse coding can be effectively used in SpBS-based sensors allowing for a sensing performance enhancement [3], although reducing the SBS threshold depending on the code length.

Compared to single-longitudinal mode lasers, such as distributed feedback (DFB) lasers, FP lasers typically provide higher optical power, which, moreover, is spectrally distributed among several longitudinal modes. These features lead to an increase in SBS threshold according to the number of modes, since the SBS threshold limits only the FP power per mode. Owing to their large spectrum, FP lasers also allow for intensity normalisation using Rayleigh-scattering (RS) measurements that are free from coherent-Rayleigh noise (CRN), thus avoiding current dithering or $\lambda$-averaging.

In this Letter, we propose and characterise the use of common multimode lasers (namely FP lasers) for simultaneous temperature and strain sensing based on both SpBS intensity and BFS measurements. To measure the SpBS components, a coherent-detection scheme is required [2], where the Brillouin signal is mixed with an optical local oscillator (OLO).

When an FP laser propagates into a fibre with a peak power per mode lower than the SBS threshold, each longitudinal mode generates SpBS components. If the FP longitudinal mode spacing is sufficiently large (greater than approximately $0.25 \mathrm{~nm}$ ) the contribution of all SpBS Stokes and anti-Stokes components can be effectively measured; a coherent-detection scheme is required, with a multi-wavelength OLO. This can actually be obtained by splitting a portion of the same FP laser light (self-heterodyne system [2]), so that the beating of each FP mode with the respective SpBS components generates an electrical signal at around the BFS.

Experiment: The experimental setup is shown in Fig. 1. To compare the performance achieved by multi- and single-longitudinal mode lasers, a DFB and an FP laser are alternatively employed. Note that, when using DFB lasers, current dithering is required to reduce CRN effects in RS measurements. However, this can be easily avoided when employing FP lasers, since their broad optical spectrum suppresses CRN effects. $10 \%$ of the CW-light of the source is used as OLO at the receiver side, while the other $90 \%$ is modulated with 127-bit Simplex-coded pulses [3] by using a Mach-Zehnder interferometer (MZI). The used DFB laser operates at $1550 \mathrm{~nm}$ and allows for a maximum power of $1.5 \mathrm{dBm}$ at the fibre input (a typical value for DFB lasers considering the actual loss between the laser and sensing fibre in Fig. 1). On the other hand, the used FP laser has a $10 \mathrm{~nm}$ linewidth, it is centred at $1545 \mathrm{~nm}$, and provides $15 \mathrm{dBm}$ at the fibre input. Pulses of $350 \mathrm{~ns}$ (allowing for $35 \mathrm{~m}$ spatial resolution) are launched into a $25 \mathrm{~km}$ standard singlemode fibre. The receiver consists of two stages: (i) a direct-detection receiver used for Rayleigh measurements which consists of $a$ pin photodiode, a transimpedance amplifier (TIA) and an analogue-digital converter (ADC) connected to a computer (PC); and (ii) a heterodyne receiver, where the OLO is mixed with the SpBS components. Signals are detected by using a $12.5 \mathrm{GHz}$ pin, followed by a TIA and a $40 \mathrm{GHz}$ electrical spectrum analyser (ESA) operating in zero-span mode. Effects of polarisation-induced fading have been reduced by decreasing the degree of polarisation of the OLO $(<1 \%)$ using a polarisation scrambler (PS).

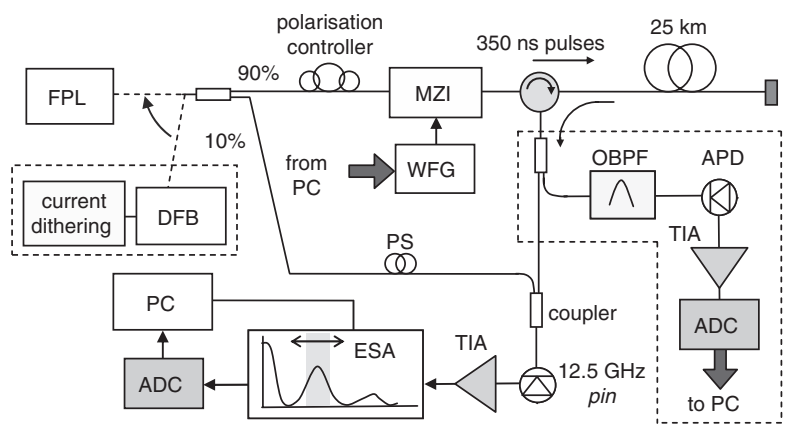

Fig. 1 Experimental setup

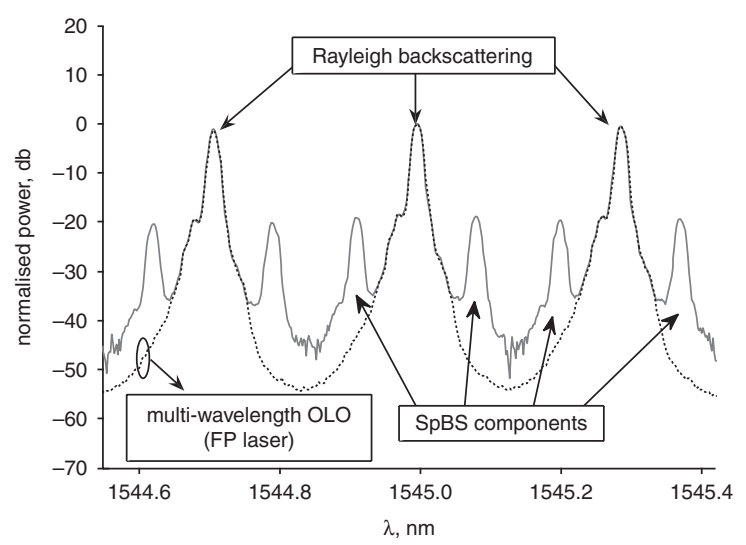

Fig. 2 Optical spectrum of three FP modes and respective Rayleigh, Brillouin Stokes and anti-Stokes components

Fig. 2 shows the spectrum of three FP longitudinal modes and the respective Rayleigh, Brillouin Stokes and anti-Stokes backscattered components. In the Figure, the power of the used FP laser and its backscattering have been normalised with respect to the peak power per mode of the laser in order to provide a clear understanding of the proposed technique. Note that the linewidth of each FP mode is narrower 
than the Brillouin spectral width, and the spectral separation among adjacent modes $(\sim 0.3 \mathrm{~nm})$ is more than twice the BFS in silica, allowing us to clearly measure the SpBS components scattered from each FP longitudinal mode.

The electrical signal from the pin represents the summed contribution of Stokes and anti-Stokes components generated by all FP longitudinal modes. This signal, at $\sim 10.9 \mathrm{GHz}$, is measured by using the ESA with a resolution bandwidth of $3 \mathrm{MHz}$ [3], which actually limits our spatial resolution to $\sim 35 \mathrm{~m}$. After acquiring $128 \mathrm{~K}$ SpBS-traces and decoding them, the BFS parameter is obtained by fitting the measured spectrum with a Lorentzian curve at each fibre position. Fig. 3 shows the measured Brillouin spectrum using the FP laser. Pump depletion has not been observed in our measurements even if the total input power $(15 \mathrm{dBm})$ is higher than the SBS threshold for singlemode lasers with coding ( $\sim 10 \mathrm{dBm}$ as reported in [3]). This is because the total power is spectrally distributed in several modes, so that the maximum peak power per mode reaches only $\sim 0 \mathrm{dBm}$ in our case. On the other hand, the SpBS intensity is obtained by simple spectrum integration, and it is used together with the BFS in (1) to compute distributed measurements, obtaining the strain and temperature resolutions shown in Fig. 4. The achieved temperature resolution, at $25 \mathrm{~km}$ distance, is reduced from $\sim 20 \mathrm{~K}$ (obtained with the DFB) down to $\sim 4.5 \mathrm{~K}$ when using the FP laser, while the strain resolution is improved from $\sim 450 \mu \varepsilon$ down to $\sim 115 \mu \varepsilon$. The potential of using standard FP lasers for performance improvement in SpBS-based sensors is evident, especially in combination with optical pulse coding and optical amplification for further improvement.

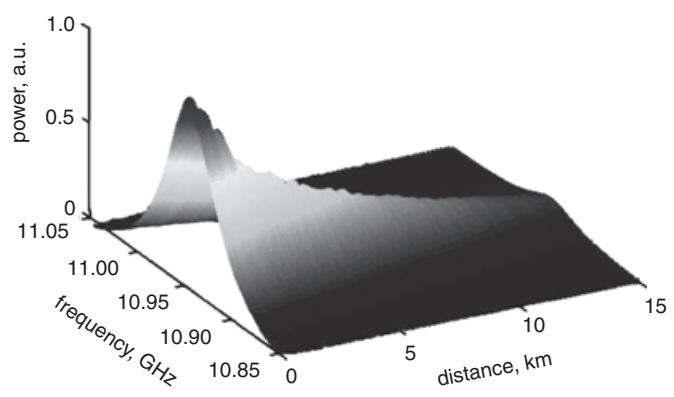

Fig. 3 Brillouin spectrum against distance
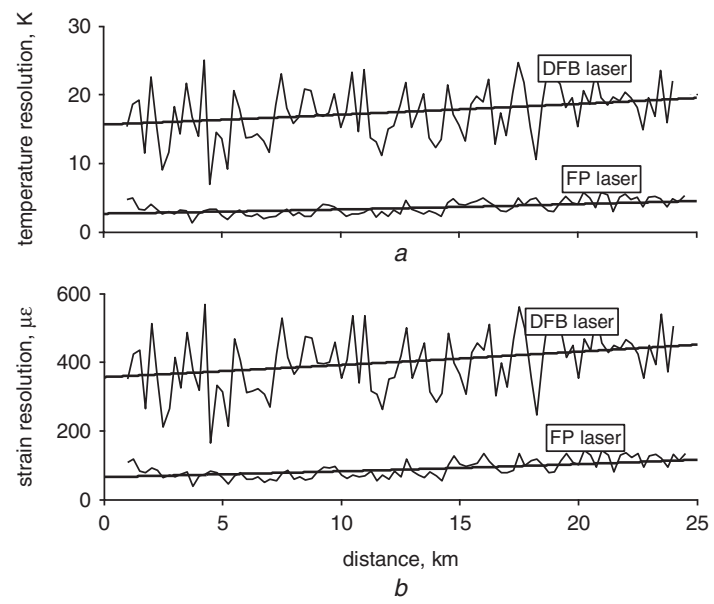

Fig. 4 Temperature resolution and strain resolution

a Temperature resolution

$b$ Strain resolution
Conclusions: We propose the use of multi-wavelength FP lasers for simultaneous strain and temperature sensing. The multi-longitudinal modes of FP lasers are characterised by a spectral power distribution which allows us to overcome the main limitations of SpBS-based sensor using singlemode lasers: higher total power can be used before reaching the limit imposed by nonlinear effects, pulse coding can be effectively used with higher peak pulse power and no coherent Rayleigh noise suppression is required when measuring the Rayleigh scattering light. We have shown that FP lasers allow us to clearly measure the Brillouin spectrum, providing a significant performance enhancement in SpBS-based sensors with respect to the typically used DFB lasers.

(C) The Institution of Engineering and Technology 2009

19 August 2009

doi: 10.1049/el.2009.2381

M.A. Soto, G. Bolognini and F. Di Pasquale (Scuola Superiore Sant'Anna, via G. Moruzzi 1, 56124 Pisa, Italy)

E-mail: m.soto@sssup.it

\section{References}

1 Bao, X., Webb, D.J., and Jackson, D.A.: 'Combined distributed temperature and strain sensor based on Brillouin loss in an optical fibre', Opt. Lett, 1994, 19, (2), pp. 141-143

2 Maughan, S.M., Kee, H.H., and Newson, T.P.: 'Simultaneous distributed fibre temperature and strain sensor using microwave coherent detection of spontaneous Brillouin backscatter', Meas. Sci. Technol., 2001, 12, pp. $834-842$

3 Soto, M.A., Bolognini, G., and Pasquale, F.Di: 'Analysis of optical pulse coding in spontaneous Brillouin-based distributed temperature sensors', Opt. Express, 2008, 16, pp. 19097-19111 